URL

\section{Optical and fluorescence microscopy}

Amnis Corporation

Andor Technology

Applied Precision

Apogee Instruments

Applied Imaging

Applied Scientific

Instrumentation

BD Biosciences Clantech

Berthold Technologies

Biomedial Photometrics

- Bioptonics

Bruker

Cambio

Carl Zeiss

Chemicon

Chroma Technology Corp

Cisbio international

Cytocell

DVC

Enamine

Energy Beam Sciences

Evident Technologies

Evrogen

Exigon

Genisphere

- Hamamatsu Photonic

Systems

Image Solutions

Intracellular Imaging

Intell ligent Imaging Innovations

Kodak

La Vision Biotec

- Leica Microsystems

Ligitlab

Lightooks

Martek Eliosciences

Mauna Kea Technologies

MBL Interna tional

Micro Luminetics

Micro Video Instruments

Nanoptek

- Nikcon Instruments

Novagen

- Olympus Life and Material

Science Europa

Omega Optical

Open Biosystems

Optical Imaging

Optronics

PCO

PerkinEImer Life Sciences

Photon Technology International

Photometrics

Polaroid

Princeton Instruments

Qbiogene

Qlmaging

- Quantum Dot Corporation

Richardson Technologies

Shimadzu North America

Thermo Bectron

Tritech Research

Unitron

Veeco

WTाec

Varian

VayTek

Vision Engineering

Fluovescent dyes

3DNA FISH probes

Optical micoscopes
ImageStream flow cytometry and cell-inaging system

$C C D$ and intens fied $C C D$ camera systems for bioimaging

Cel Woldx for imaging Iving cells SoftWoRx Explorer 5D image viewer

XON electron-mulfplying (EMO CCD camera and low-light imaging systems

Imaging systems and image analysis for cytogenetics and pathology

Automated systems for micoscopy and fuarescence screening

Fucrescent proteinș reagents for molecular and cell biblogy

Nght OWL imaging system for microscopic and macroscopic luminescent and fluorescent signals

MACROscope wide-field confocal laser-scanning microscopes

Optical projection tomography (OPT) scanning service

Automated platiorms for mass spectrometry NMR, EPR, and magnefic resonance imaging

STARFISH chromosome paints reselers of specialized biochemicals

LSM 5 LNE confocal laser-scanning microscopes stereomicosopes, upright micoscopes,

inverted micoscopes, fucrescence correlation spedrascopy

Fuorescent proteinș antibodies

Optical filters and fluovescence filter sets

Reagents and kits for TR-FRET (time-resolved fluovescence resonance energy transfer) assays

Ohromaprobe Multiprobe system for fucrescent in stu hybridzation

Digital cameras for light microscopy, image-analysis software

Products for light microscopy and election microscopy

Indium gallum phosphide MP-T2 EvTags and Biotin Evifluo quantum dot fluorescence labels

Products for genomics and proteorics research, fluorescent proteins

Fuarescence-tageed UNA aliganucleofides

Digital imaging systerns and cameras

Microscopes and imaging systems

Digital fluarescence and photometry systems

Digital microscopy workstations and imaging softwase

Image Station in wro F and FX multi-mode imaging systerns, image-analysis softwase

TiMScope laser-scanning micoscope, fuorescence-lifetime microscopy system

TCS 4P fluorescence confocal imaging system, DM digtal microscope range, micoscope systems

Optical coherence tamagraphy (OCT) development

Whole mouse imaging, Pan-A-See-Ya panaramic imaging system

Fuarescent tags based on algalproteins

Miniaturized fbre-optic fuarescence confocal microscopy system for in wivo intra-tissue imaging

Caral Hue range of fluarescent proteins

Cyocam cooled CCD cameras forlowlight imaging

Microscopes and accessories

Digital photon-tunneling microscope with near-field optics

Digital Eclipse C1 Spectral Imaging confocal system, microscopes

Reagents and kits for molecular biblogy, Sensilight fluorescent reagents

Digital microscopes wide-field micoscopes, Fluoview confocal

laser-scanning microscopes cell "range of imaging stations

Optical filters and fluovescence filter sets, microscopes

STAR*FISH dramosome paints

Brain and retinal imaging systems

Digital microscope cameras image-analysis softwase

Digital video camera systerns

UtraVlew confocal laser-scanning microscope system

Imagemaster fuovescence and general imaging systems

$C C D$ and EMCCD cameras

Digital imaging systems for microscopes

Equipmentfor imaging and spectroscopy

Fuorescent proteins and fusion vectors, products for FISH

High-performance digtal cameras and RGB fitters for micoscopy

Qdots quantum dotfluorescent tags, Mosaicgene expression assay system

RTM 30 microscope system, hightresolution field microscope

AM 8800 automated Fourier transform infraed micoscope

Inflared and Raman microscopes laser sources, image-analysis software

Dissecting stereomicoscope system, microscope accesscries

Scanning-probe microscopes

Optical and scanning-probe microscopes

Spedrophotometers, infrared microscopes and imaging systems

Digital imaging systerns, camers, imaging software

Microscopes microscope accessories
Seattle, Washington

Belfast UK

ksaquah, Washington

Rosevile, Calfomí

San Jose, Calfornia

Eugene, Oregon

Mountain View Callifornia

Bad Wildoad Germany

Waterloo, Ontario

Edinburght UK

The Woodlands Texas

Cambridge UK

Jena,Germany

Temecula, Calfornia

Brattlebow, Vermont

Bagnols-sur-Cère, France

Cambridge, UK

Austin, Texas

Kiev, Ulraine

East Granby Connecticut

Troy New Yark

Moscow, Russia

Vedbaek, Denmak

Hatfield, Pennsylvania

Hamamatsu City, Japan

Preston, UK

Cinchnati, Ohio

Dermer, Colorado

Rochester, New York

Bielefeld, Germany

Wetzlax, Germany

Westiord, Massachusetts

Enónitas, Calfornia

Columbia, Maryland

Paris, France

Woburn, Massachusetts

Los Angeles, Callfomia

Awon, Massachusetts

Concord, Massaciusetts

Melvile, New York

Madison, Wisconsin

Hamburg Germany

Brattlebow, Vermant

Huntsvile, Alabama

Rehowot lsrael

Goleta Calfornia

Kelheim Germany

Boston, Massachusetts

Birmingham, New lessey

Tucson, Arizona

Waltham, Massachusetts

Trenton, New lersey

Invine Calfornia

Bumaby Brtish Columbia

Hayward, Californa

Bolton Ontario

Columbia Maryland

Waltham Massachusetts

Los Angeles, Calfornia

Bchemia, New York

Woodbury, New Yok

Ulim, Germany

Palo Atta, Calfomí

Faifield, lowa

Woking UK

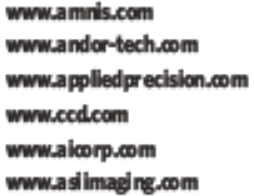

www.as imading com

www.clontech.com/tlontech

wmw.bertholdtech.com

mmw.confocalcom

mumbibioptonics.com

mumbrukeeccom

wuw.camblacoulk

mwwizelss.com

muwschemican.oom

wmwichromacom

wmwhtrf-assays.com

mumcytocellcom

mundecos.com

www.enamine-net

www.ebsciences.com

www.evidenttech_om

mmw.evrogencom

mmisexiponcom

wmw.genispherecom

muwh hamamatsi.com

muwdimsolcoulk

mwwintracellular.com

wwwintelligent-Imaging.com

wmwkodakcom

mwwlavisionblotec de

mundeica-microsystems.com

wwwlightlabimaging.com

mwwlil ghtool scom

muwumartekblacom

mww.maunakeatech.com

www.mblintloom

mun.cryocam.oom

muwumvilinc.com

mwwrnanoptekcom

www.nilkon.com

www.novagen.oom

mww.olympus.com

mwwsomegafilters.com

mww.openblosystems.com

www.opt-Imagingeom

mmwioptronics.com

mmw.pcade

las.perkinel mexcom

mum.ptinjerm

wmwiphotomet.com

mmw.polarold.com

mww.princetoninstrumentscom

mwurqulogenecom

mww.qimaging.com

wmwiqdots.com

mwwrichardson-techcom

mww.sslishimadaucom

www.thermacom

www.tritechresearchcom

mmwurit tronusacom

mwiviveeco.com

mmw.wittecide

mww.variarinc.com

mmw.vaytekcom

mww.visioneng.omm 


\begin{tabular}{|c|c|c|c|}
\hline COMPANY & PRODUCTS/ACTIVITY & LOCATION & URL \\
\hline Vysis & Chromosome paints for FISH & Downer's Grove, Eingis & wwwyysis.com \\
\hline Xenogen & Luciferase-based in wivo imaging for live animals & Alameda Cal'fomia & wwwsenogen_com \\
\hline \multicolumn{4}{|c|}{ Laser scanners and imaging systems } \\
\hline Affymetrix & GeneChip mioroanay systems and scamess & Santa Clara California & www affymetrixcom \\
\hline Agilent & Catalogue and custom DNA microarrays, scanner and software & Palo Alb, Califomia & www agllertcom \\
\hline Alpha Innotech & Alpha Array microarray reader & San Leandra California & www alphainnotechcom \\
\hline Applied Biosystems & DNA sequencers protein sequencers mass spectrometers, chromatography systems & Foster City Calfornia & homeappliedblosystems.com \\
\hline Beckman Coulter & Automated tools formolecular biology, biochemistry, genomics and proteomics & Fullerton, California & www beckmancoulter.com \\
\hline - Bio-Rad & Imaging systerns for molecular biology & Hercules, Califomia & www blo-radcom \\
\hline BMG Labtech & Scanners, microarray readers and handing systerns & Offenburg, Germany & wwwlbing-labtechnologles_com \\
\hline Cellomics & AnayScan HCS and KineticScan HCS systerns for automaled cell-based high-content screening & Pittsburgh, Pennsylvania & wwwecel lomics.com \\
\hline CyBio & Pipetting, Iiquid handling incubation, and imaging systerns, CyEio Nanoscan platereaders & Jeną Germany & wwweyblo-agcom \\
\hline Cyntellect & High-throughput LEAP laser-scanning and image-analysis system for cellular analysis & San Diega Callornla & wwwcyntellect.com \\
\hline Fuifilim & Imaging systems for protein electrophoresis and array analysis & Stamford Connecficut & wwwtulimed_com \\
\hline - GE Healthcare & IN Cell confocal imaging system for cell screening scamers and analysis software & Chalfont St Gles UK & wwwamershamoouk \\
\hline Genetix & Q-bot scanners & New Miton, UK & www genethicoulk \\
\hline LH-COR Biosciences & Infrared imaging system for western blot and in-cell western blot assay analysis & Lincoln, Nebraska & www licotcom \\
\hline Metrigenix & 4D Assay scamer for DNA microarrays & Gaithersburg, Maryland & www metrigenkxcom \\
\hline Miraililo & CRB1O lie scannes, image-analys's software & Alameda Califomia & www mi ralliacom \\
\hline Molecular Devices & GenePix $4000 B$ micoana y scanner and analysis software, ImageExpress for live-cell assays & Foster City, Calfornia & www moleculardevices.com \\
\hline UYP & Bioimaging systems for gels, microarrays and microplates & Upland Calfornia & www unp.com \\
\hline
\end{tabular}

\begin{tabular}{ll}
\hline Image-analysis software & \multicolumn{1}{l}{ CellMine image-analysis software for high-content screening } \\
Biolmagene & Image-processing software \\
Biplane & Image-analysis software \\
Clemex & Cellenger image-analysis software \\
Definiens & 3D imaging software \\
Improvision & ImagePro imaging software \\
Media Cybernetics & Image-analysis software \\
Mirew & IPLab scientific imaging software \\
Scanalytics & IGOR image-processing and analysis software \\
Wavemetrics &
\end{tabular}

San Matea, California
Zörich, Switzerland
Longueuil, Quebec
Munich, Germany
Coventry UK
Silver Spring Maryland
Gyeongzi-da, South Korea
Rodkile, Maryland
Portand, Oregon

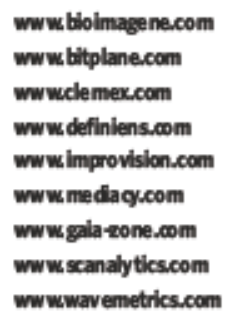

\begin{tabular}{|c|c|c|c|}
\hline \multicolumn{4}{|l|}{ General } \\
\hline Adive Motif & Kits and reagents for nucleicaoid isolation, cell fractionation and gene slencing & Carsbad Calfomia & www acthemoti icom \\
\hline Aetos & Biopolymer for preserving and transporting live specimens & Ophelka, Alabama & wwwaetos.com. \\
\hline Andurus & Laser-capture dissection, microgenomics, RNA amplification & Mountain View, Califomia & wwwarctur.com \\
\hline Biognostik & Antisense oligonucleofide synthesis kits and services & Gottingen, Gemany & www blognostikcom \\
\hline BOMOL & Biochernicals for life-science research & Pymouth Meefing Pernsylvanla & www blomolcom \\
\hline Biosearch Technologies & Fuorophores and quenchers, Black Hole Quencher & Novata, Calfornia & www blosearchitech.com \\
\hline Cambrex & Products for molecular and cell biology research & Walkersvile, Maryland & wwwcambrexcom \\
\hline Cayman Chemical & Antibodies, proteins and small biological molecules & Ann Aubor, Michigan & wwwcaymanchemcom \\
\hline EMD Biosciences & Novabiochem, Calbiochem, Novagen and Oncogene Research produds & San Diega California & splashemdblosciences.com \\
\hline Enzo Life Sciences & Nonradioactive labelling and detection reagents for molecular biobgy & Farmingdale, New York & wwwerzolifesdences:com \\
\hline HORIBA Jobin Yvan & Fluarescence spedrophotomers & Kyota, lapan & wwwjobinywon_cosk \\
\hline Eppendorf & Laboratory instrumentation and consumables for molecular and œll biology & Hamburg Germany & wwweppendorf.com \\
\hline - Genscript & Kits and services for molecular biology, oliganu cleotide probes & Piscataway, New Jersey & wwwgenseriptcom \\
\hline Hamilton Compary & Automation for the life-sciences & Reno, Nevada & wwwhamiltoncompcom \\
\hline IBA & Olganucleofide probes & Gottingen, Gemmany & wwwilbo-gacom \\
\hline KPL & Nucleic-acid labeling and detection kits & Gaithersburg, Maryland & wwwinww kpl.com \\
\hline Invitiogen & Kits and reagents for genornics proteornics, molecular and cell biology & Carkbad, Calfornia & wwwimitrogencom \\
\hline Molecular Beacons & Molecular beacon probes for in situ RNA detection & Newark, New Jersey & www molecular-beacons.org \\
\hline $\begin{array}{l}\text { Plerce Chemical/ } \\
\text { Pierce Biotechnology }\end{array}$ & Products for molecular biology, cell biology, immunology and chromatography & Rockford, Finois & www plercenetcom \\
\hline Promega & Vectors reagents and lits for genornics, proteornics and cell biology & Madison, Wiscansin & www promega_com \\
\hline Qiagen & Automated workstations kits and reagents for genomics, proteornics, molecular and cell biology & Valencia Califomia & wwwq qagen_com \\
\hline - REDSystems & Antibodies, fluorescence-based cytokine detection kts & Minneapols, Minnesota & wwwRnDSystems.com \\
\hline Roche Applied Science & Instrumentation and reagents for PCR & Lewes, UK & www roche-applied-sclence.com \\
\hline - Sigma-Aldrich & Reagents and biochernicals for life sciences research & St Louis Missouri & sigma-aldrichcom \\
\hline - Stratagene & Kits and reagents for genornics and molecular biology & La Jola California & www stratagenecom \\
\hline - Takara Bio & Reagents, kits and custom services for molecular biology, genomics, proteornics research & Shiga, lapan & wwwtakara-blacom \\
\hline Tecan & Laboratory automation for the life solences & Mănnedort, Switzerland & wwwetecancom \\
\hline Upstate & Kitș reagents and services for cell signalling neuroscience and cell biology & Charlottesvile, Vrginia & wwwupstatecom \\
\hline USB & Reagents formolecular and cell biobgy & Ceveland Ohio & www usbuebcom \\
\hline
\end{tabular}

